\title{
Filtration in porous rock with initial deposit
}

\author{
Yuri Osipov* and Yuri Galaguz \\ Moscow State University of Civil Engineering, Yaroslavskoe shosse, 26, Moscow, 129337, Russia
}

\begin{abstract}
The problems of underground fluid mechanics play an important role in the design and preparation for the construction of tunnels and underground structures. To strengthen the insecure soil a grout solution is pumped under pressure in the porous rock. The liquid solution filters in the pores of the rock and strengthens the soil after hardening. A macroscopic model of deep bed filtration of a monodisperse suspension in a porous medium with a size-exclusion mechanism for the suspended particles capture in the absence of mobilization of retained particles is considered. The solids are transported by the carrier fluid through large pores and get stuck at the inlet of small pores. It is assumed that the accessibility factor of pores and the fractional flow of particles depend on the concentration of the retained particles, and at the initial moment the porous medium contains an unevenly distributed deposit. The latter assumption leads to inhomogeneity of the porous medium. A quasilinear hyperbolic system of two first-order equations serves as a mathematical model of the problem. The aim of the work is to obtain the asymptotic solution near the moving curvilinear boundary - the concentration front of suspended particles of the suspension. To obtain a solution to the problem, methods of nonlinear asymptotic analysis are used. The asymptotic solution is based on a small-time parameter, measured from the moment of the concentration front passage at each point of the porous medium. The terms of the asymptotics are determined explicitly from a recurrent system of ordinary differential and algebraic equations. The numerical calculation is performed by the finite difference method using an explicit TVD scheme. Calculations for three types of microscopic suspended particles show that the asymptotics is close to the solution of the problem. The time interval of applicability of the asymptotic solution is determined on the basis of numerical calculation. The constructed asymptotics, which explicitly determines the dependence on the parameters of the system, allows to plan experiments and reduce the amount of laboratory research.
\end{abstract}

\section{Introduction}

The study of suspension filtration in a porous medium is an important problem for many areas of science and technology. The filtration process is accompanied by the formation of deposit in the pores, which changes the structure and properties of the porous medium [1]. During the construction of underground facilities for the storage of hazardous waste, it is

\footnotetext{
* Corresponding author: yuri-osipov@mail.ru
} 
necessary to reinforce the soil and create watertight partitions to protect structures against groundwater. Liquid grout solution is pumped into the soil under pressure. Grains of the solution are retained in the pores of the rock and create a durable waterproof layer during solidification [2].

When solid suspended particles are transported by the fluid flow, some particles are retained on the framework of the porous medium. Depending on the properties of the suspension and the porous medium, mechanical interaction, diffusion, viscosity, electrostatic and gravitational forces can play an important role in the capture of particles [3]. If the sizes of particles and pores are of the same order, then in many cases the sizeexclusion mechanism of particle capture prevails: solid particles freely pass through pores of large diameter and get stuck in pores whose diameter is smaller than the particle size [4]. The complex topology of the porous channels and the varying sizes of the pore channels cause particles to be trapped at the narrowing of the pores far from the filter inlet. With deep bed filtration, deposit is formed in the entire porous medium, and not only in its surface layer. When injected at the porous medium inlet, some suspended particles are transported by the fluid flow through the porous sample and leave the porous medium at the outlet, others get stuck in narrow pores and form a deposit.

A traditional mathematical model for a one-dimensional deep bed filtration of an incompressible monodisperse suspension in a porous medium with size-exclusion mechanism for trapping particles determines the concentrations of suspended and retained particles by a system of two partial differential equations. The mass balance equation for suspended and retained particles is an analogue of the continuity equation; the kinetic equation determines the deposit growth rate [5]. More sophisticated filtration models for particles and pores of various sizes, constructed on the basis of the balance of suspended and retained particles, are given in [6].

Exact solutions of the filtration problem with pores completely accessible to suspended particles were obtained in [7]; in this case, the characteristics of the system are straight lines.

The introduction of the pore accessibility factor and the particle flux reduction ratio (fractional flow) leads to curvilinear characteristics. Exact solutions become much more complex [8]. Asymptotic solutions that use the distance to the concentration front as a small parameter, are applicable for the time ranges of filtration processes encountered in laboratory and field studies.

Standard filtration models with a pure porous framework suggest that at the initial moment there are no suspended and retained particles in the porous medium. In this paper, a more general case is considered: a porous medium contains an initial deposit and clear water before the start of filtration. The studied model describes the filtration of the suspension when changing the suspension flow and the reverse flow of pure water. The deposit accumulates in a porous medium with the filtration of a direct suspension stream. When water is pumped back, mobilization and leaching of retained particles occur [9].

It is assumed below that when a suspension is displaced by pure water moving in the opposite direction, an incomplete mobilization of particles occurs, and the retained particles are partially preserved in a porous medium. An unevenly distributed initial deposit affects the filtration process when the suspension is injected into the porous medium. An asymptotic and numerical solution of the problem with initial deposit for a small limiting deposit was obtained in [10]. However, in the general case, analytical solutions to the filtration problem for a suspension in a porous medium with an initial deposit are not available.

In this paper, the filtration of a suspension that displaces pure water from a porous medium with an initial deposit is considered. The suspended particles concentration front moves in the porous medium with variable velocity. The dependence of the initial deposit 
on the coordinate in the model, which takes into account the dependence of porosity and fractional flow on the accumulated deposit, entails the curvilinearity of the front. An asymptotic solution is constructed near the concentration front with a small parameter equal to the time after the passage of the front. The results of numerical simulation show that the asymptotics is close to a solution over a large time range.

\section{Filtration model}

The model assumes an insignificant diffusion / dispersion. Small concentrations of suspended and retained particles do not affect the volume balance of the total flow [11]. The filtration equation is applicable to filtration, the capture of particles in the orifices of small pores and the retention of small particles [12]. The model uses only the size-exclusion capture mechanism of individual particles and excludes the formation of arch bridges at the entrance of large pores [13]. The rate of deposit formation is proportional to the advective particle flow; the retained particles do not detach from the framework of the porous medium. It is assumed that the flow is single-phase, the suspension injected into the porous medium contains the same water as the formation water, which saturates the rock first.

The above assumptions imply a system of equations of deep bed filtration, consisting of the mass balance equation for the concentrations of suspended and retained particles and the kinetic equation for the deposit growth rate. In dimensionless variables, the equations take the form

$$
\begin{aligned}
& \frac{\partial(g(S) C)}{\partial t}+\frac{\partial(f(S) C)}{\partial x}+\frac{\partial S}{\partial t}=0 \\
& \frac{\partial S}{\partial t}=\Lambda(S) C .
\end{aligned}
$$

Here $C(x, t), S(x, t)$ are the dimensionless concentrations of suspended and retained particles; the accessibility porosity coefficient $g(S)$, the fractional flow through accessible pores $f(S)$ and the filtration coefficient $\Lambda(S)$ are given positive continuous differentiable functions.

It is assumed that a suspension with a constant concentration of suspended particles is injected at the porous medium inlet $x=0$; at the initial time $t=0$, there are no suspended particles in the porous medium and a deposit $s_{0}(x)$ is unevenly distributed over the porous sample. The corresponding initial and boundary conditions are

$$
\begin{aligned}
& \left.C\right|_{x=0}=1 ; \\
& \left.C\right|_{t=0}=0 ; \\
& \left.S\right|_{t=0}=s_{0}(x),
\end{aligned}
$$

where $s_{0}(x)$ is a non-negative continuous differentiable function.

Conditions (3) - (5) determine the unique solution to the problem in the domain $\Omega=\{(x, t): 0 \leq x \leq 1, t \geq 0\}$. The solution has a discontinuity, since conditions (3) and (4) are not consistent at the origin. The break line $\Gamma$ is given by the formula

$$
t_{\Gamma}(x)=\int_{0}^{x} \frac{g\left(s_{0}(x)\right)}{f\left(s_{0}(x)\right)} d x .
$$

The curve $\Gamma$ divides the domain $\Omega$ into two subdomains 
$\Omega_{0}=\{(x, t): 0 \leq x \leq 1,0 \leq t<x\}, \Omega_{S}=\{(x, t): 0 \leq x \leq 1, t \geq x\}$.

Filtration of the suspension occurs in the domain $\Omega_{S}$; in the domain $\Omega_{0}$, there are no suspended particles and the retained particles concentration does not depend on time. The concentration front of suspended particles spreads in a porous medium along the line $\Gamma$ with a velocity

$$
v=\frac{f\left(s_{0}(x)\right)}{g\left(s_{0}(x)\right)} .
$$

The solution of problem (1) - (5) is constant in the domain $\Omega_{0}:\left.C(x, t)\right|_{(x, t) \in \Omega_{0}}=0$, $\left.S(x, t)\right|_{(x, t) \in \Omega_{0}}=S_{0}(x)$. In the domain $\Omega_{S}$, the solution is positive: $\left.C(x, t)\right|_{(x, t) \in \Omega_{S}}>0$, $\left.S(x, t)\right|_{(x, t) \in \Omega_{S}}>s_{0}(x)$. Below, the problem (1) - (5) is considered on the boundary $\Gamma$ and in its neighborhood in the domain $\Omega_{S}$.

\section{Asymptotic solution near the concentration front}

Assume that the coefficients of system (1), (2) can be expanded in Taylor series with coefficients depending on $x$ :

$$
\begin{gathered}
g(S)=g_{0}+g_{1}\left(S-s_{0}\right)+\frac{g_{2}}{2}\left(S-s_{0}\right)^{2}+\frac{g_{3}}{6}\left(S-s_{0}\right)^{3}+O\left(S-s_{0}\right)^{4} \\
f(S)=f_{0}+f_{1}\left(S-s_{0}\right)+\frac{f_{2}}{2}\left(S-s_{0}\right)^{2}+\frac{f_{3}}{6}\left(S-s_{0}\right)^{3}+O\left(S-s_{0}\right)^{4} \\
\Lambda(S)=\Lambda_{0}+\Lambda_{1}\left(S-s_{0}\right)+\frac{\Lambda_{2}}{2}\left(S-s_{0}\right)^{2}+O\left(S-s_{0}\right)^{3}
\end{gathered}
$$

Here, the index 0 means the value of the function for $S=s_{0}(x)$, the nonzero index $j$ is the corresponding derivative of order $j$ with $S=s_{0}(x)$.

In the domain $\Omega_{S}$ in the vicinity of the concentration front $\Gamma$, an asymptotic solution is constructed in a form:

$$
\begin{aligned}
& S(x, t)=s_{0}(x)+\sum_{i=1}^{n} \frac{\left(t-t_{\Gamma}(x)\right)^{i}}{i !} s_{i}(x)+O\left(t-t_{\Gamma}(x)\right)^{n+1} \\
& C(x, t)=c_{0}(x)+\sum_{i=1}^{n} \frac{\left(t-t_{\Gamma}(x)\right)^{i}}{i !} c_{i}(x)+O\left(t-t_{\Gamma}(x)\right)^{n+1} .
\end{aligned}
$$

Expansions (9), (10) represent the solution of a system of two partial differential equations, depending on two variables, in the form of Taylor series in powers of the small parameter $t-t_{\Gamma}(x)$. The coefficients of the series depend on one variable $x$ and satisfy the system of first-order ordinary differential equations. Asymptotics is a local analytic solution in an explicit form obtained with the required accuracy.

Substituting the expansions (8) - (10) into equations (1), (2) and equate the terms with the same powers $\left(t-t_{\Gamma}(x)\right)$ gives algebraic relations for the first terms of the asymptotics

$$
s_{1}=\Lambda_{0} c_{0}
$$




$$
\begin{aligned}
& s_{2}=\Lambda_{0} c_{1}+\Lambda_{0} \Lambda_{1} c_{0}^{2} \\
& s_{3}=\Lambda_{0} c_{2}+3 \Lambda_{0} \Lambda_{1} c_{0} c_{1}+\left(\Lambda_{2} \Lambda_{0}+\Lambda_{1}^{2}\right) \Lambda_{0} c_{0}^{3},
\end{aligned}
$$

and a recurrent system of differential equations

$$
\begin{gathered}
\left(f_{0} c_{0}\right)^{\prime}+\Lambda_{0} c_{0}+\beta \Lambda_{0} c_{0}^{2}=0 \\
\left(f_{0} c_{1}\right)^{\prime}+\Lambda_{0}\left(3 \beta c_{0}+1\right) c_{1}+\left(\beta \Lambda_{1}+\gamma \Lambda_{0}\right) \Lambda_{0} c_{0}^{3}+\left(f_{1} \Lambda_{0} c_{0}^{2}\right)^{\prime}+\Lambda_{0} \Lambda_{1} c_{0}^{2}=0 \\
\left(f_{0} c_{2}\right)^{\prime}+\Lambda_{0}\left(4 \beta c_{0}+1\right) c_{2}+\left(f_{2} \Lambda_{0}^{2} c_{0}^{3}\right)^{\prime}+\left(f_{1} \Lambda_{0} \Lambda_{1} c_{0}^{3}\right)^{\prime}+3\left(f_{1} \Lambda_{0} c_{0} c_{1}\right)^{\prime}+\left(\Lambda_{0} \Lambda_{1}^{2}+\Lambda_{0}^{2} \Lambda_{2}\right) c_{0}^{3}+ \\
+\left(\beta \Lambda_{0}^{2} \Lambda_{2}+\beta \Lambda_{0} \Lambda_{1}^{2}+3 \gamma \Lambda_{0}^{2} \Lambda_{1}+\delta \Lambda_{0}^{3}\right) c_{0}^{4}+\left(3 \Lambda_{0} \Lambda_{1}+6 \gamma \Lambda_{0}^{2} c_{0}+6 \beta \Lambda_{0} \Lambda_{1} c_{0}\right) c_{0} c_{1}+3 \beta \Lambda_{0} c_{1}^{2}=0
\end{gathered}
$$

Here $\alpha=g_{0} / f_{0}, \beta=g_{1}-\alpha f_{1}, \gamma=g_{2}-\alpha f_{2}, \delta=g_{3}-\alpha f_{3}$.

From formula (3), the conditions for the unique solutions of ordinary differential equations (13)-(15) are obtained:

$$
\left.c_{0}\right|_{x=0}=1 ;\left.\quad c_{1}\right|_{x=0}=0 ;\left.\quad c_{2}\right|_{x=0}=0 .
$$

The equations and initial conditions for the next asymptotic terms are constructed similarly. The Bernoulli equation (13) for the main term of the asymptotic solution determines the suspended particles concentration on the concentration front.

Solving equations (11) - (15) and substituting the solutions in expansions (9), (10) gives the asymptotics of problem (1) - (5) in the domain $\Omega_{S}$.

For a constant initial deposit $s_{0}=k=$ const, the expansion coefficients (8) do not depend on $x$. The asymptotic solution near the concentration front $t=t_{\Gamma}(x)$ has the form

$$
\begin{aligned}
& C(x, t)=\frac{1}{(1+\beta) e^{\frac{\Lambda_{0}}{f_{0}} x}-\beta}+\frac{\left(\frac{1}{2} \gamma \Lambda_{0}\left(1+e^{\frac{\Lambda_{0}}{f_{0}} x}\right)+(1+\beta) \sigma e^{\frac{\Lambda_{0}}{f_{0}} x}\right)\left(1-e^{\frac{\Lambda_{0}}{f_{0}} x}\right)}{\left((1+\beta) e^{\frac{\Lambda_{0}}{f_{0}} x}-\beta\right)^{3}}\left(t-t_{\Gamma}(x)\right)+O\left(t-t_{\Gamma}(x)\right)^{2} \\
& S(x, t)=s_{0}(x)+\frac{\Lambda_{0}}{(1+\beta) e^{\frac{\Lambda_{0}}{f_{0}} x}-\beta}\left(t-t_{\Gamma}(x)\right)+ \\
& +\Lambda_{0} \frac{\left(\frac{1}{2} \gamma \Lambda_{0}\left(1+e^{\frac{\Lambda_{0}}{f_{0}} x}\right)+(1+\beta) \sigma e^{\frac{\Lambda_{0}}{f_{0}} x}\right)\left(1-e^{\frac{\Lambda_{0}}{f_{0}} x}\right)+\Lambda_{1}\left((1+\beta) e^{\frac{\Lambda_{0}}{f_{0}} x}-\beta\right)}{\left((1+\beta) e^{\frac{\Lambda_{0}}{f_{0}} x}-\beta\right)^{3}}\left(t-t_{\Gamma}(x)\right)^{2}+O\left(t-t_{\Gamma}(x)\right)^{3} .
\end{aligned}
$$

\section{Numerical simulation}

For the calculations, the values of the variable coefficients (8) for particles of three sizes, calculated on the basis of experimental data are used [14] (see table 1). 
Table 1.

\begin{tabular}{cl}
\hline Particle radius, um & \multicolumn{1}{c}{ Equation coefficients } \\
\hline & $g(S)=0.9987+9.1 \cdot 10^{-13} S-3.73 \cdot 10^{-8} S^{2}+6.1 \cdot 10^{-5} S^{3}$ \\
$r_{1}=1.5675$ & $f(S)=0.9999+1.8 \cdot 10^{-5} S-2.05 \cdot 10^{-7} S^{2}+2.848 \cdot 10^{-4} S^{3}$ \\
& $\Lambda(S)=0.11-0.01351 \cdot S+4.49 \cdot 10^{-5} S^{2}+1.163 \cdot 10^{-3} S^{3}$ \\
\hline & $g(S)=0.9743-8.88 \cdot 10^{-14} S+1.27 \cdot 10^{-11} S^{2}-1.24 \cdot 10^{-9} S^{3}$ \\
& $f(S)=0.9947+6.27 \cdot 10^{-5} S-2.9 \cdot 10^{-8} S^{2}+6.21 \cdot 10^{-10} S^{3}$ \\
$r_{2}=2.179$ & $\Lambda(S)=0.51-5.956 \cdot 10^{-3} S+2.29 \cdot 10^{-6} S^{2}+1.35 \cdot 10^{-8} S^{3}$ \\
& $g(S)=0.7635+2.44 \cdot 10^{-15} S+3.2 \cdot 10^{-14} S^{2}+3.6 \cdot 10^{-13} S^{3}$ \\
& $f(S)=0.9075+2.315 \cdot 10^{-4} S+2.27 \cdot 10^{-8} S^{2}-3.42 \cdot 10^{-8} S^{3}$ \\
$r_{3}=3.168$ & $\Lambda(S)=1.551-3.467 \cdot 10^{-3} S-1.16 \cdot 10^{-6} S^{2}-1.16 \cdot 10^{-7} S^{3}$ \\
\end{tabular}

The numerical calculation of the problem was performed by the finite difference method using an explicit TVD scheme with the superbee limiter function $[15,16]$ for the nonlinear distribution of the initial deposit

$$
s_{0}(x)=\frac{1+(x-2)^{2}}{5} .
$$

The relationship between the time and coordinate steps for the filtration problem is chosen according to the Courant convergence condition [17].

In the figures 1-4 asymptotics is indicated by dashed and dotted lines, and the numerical solution is drawn by solid lines.

Graphs of the concentration fronts for particles of three types are presented in figure 1.

Due to the smallness of the coefficients with powers of $S$ (see table 1), all three lines do not differ much from the straight lines given by the formula $t=\alpha x$.

Figures 2-4 show the graphs of suspended and retained concentrations for three types of particles depending on the coordinate at time $t=0.5$ and on time at the porous medium outlet at $x=1$.

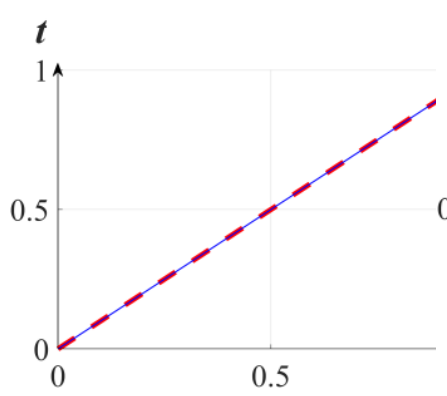

a

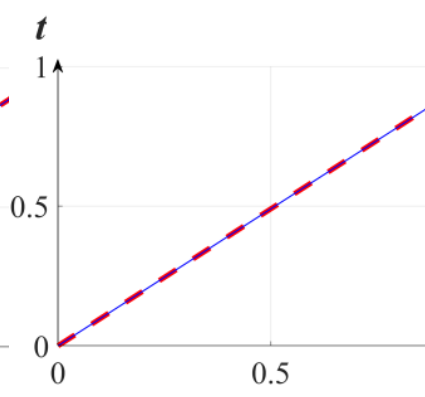

$\mathrm{b}$

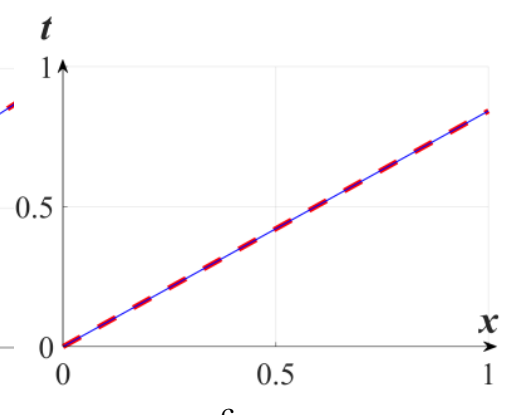

c

Fig.1. Concentration fronts a) $r_{1}=1.5675$, b) $r_{2}=2.179$, c) $r_{3}=3.168$. 


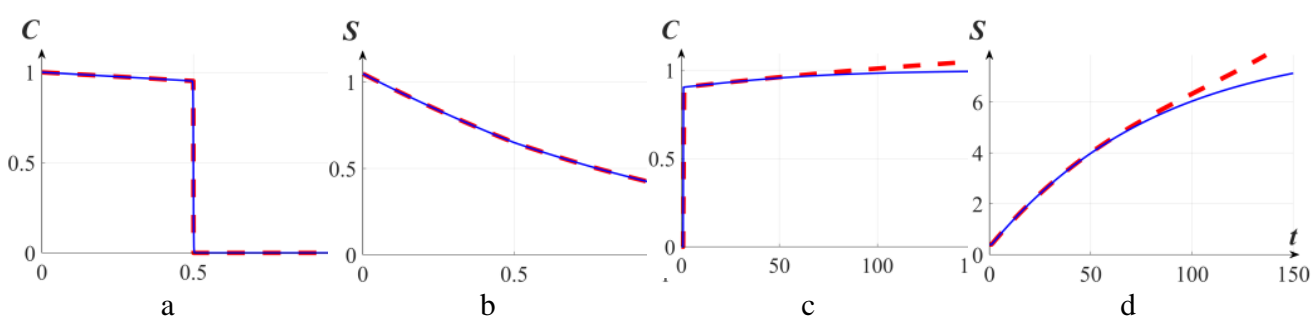

Fig. 2. Particles of radius $r_{l}=1.5675$ a) $\left.C(x, t)\right|_{t=0,5}$, b) $\left.S(x, t)\right|_{t=0,5}$, c) $\left.C(x, t)\right|_{x=1}$, d)

$$
\left.S(x, t)\right|_{x=1} \text {. }
$$

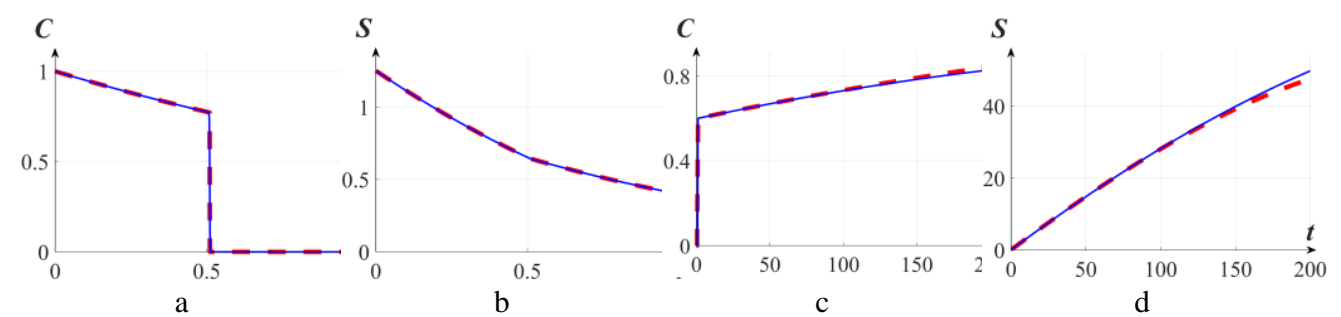

Fig. 3. Particles of radius $r_{2}=2.179$ a) $\left.C(x, t)\right|_{t=0,5}$, b) $\left.S(x, t)\right|_{t=0,5}$, c) $\left.C(x, t)\right|_{x=1}$, d)

$$
\left.S(x, t)\right|_{x=1} .
$$
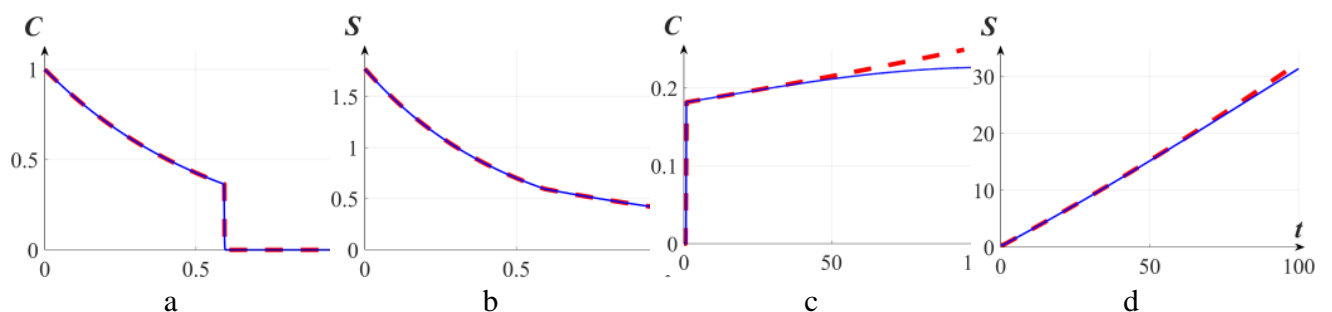

Fig. 4. Particles of radius $r_{3}=3.168$ a) $\left.C(x, t)\right|_{t=0,5}$, b) $\left.S(x, t)\right|_{t=0,5}$, c) $\left.C(x, t)\right|_{x=1}$, d)

$$
\left.S(x, t)\right|_{x=1} .
$$

At $t=0.5$, the graphs of the asymptotics and the numerical solution practically coincide. At the moment $t=0.5$ on the concentration front the graphs of suspended particles concentration are discontinuous (figures $2 \mathrm{a}, 3 \mathrm{a}, 4 \mathrm{a}$ ), and the graphs of the retained particles concentration have a kink (figures $2 \mathrm{~b}, 3 \mathrm{~b}, 4 \mathrm{~b}$ ). Break and kink points are determined by the relation (6) at $t_{\Gamma}(x)=0.5$ for the initial deposit (17).

Graphs of concentrations at $x=1$ (figures $2 \mathrm{c}, \mathrm{d}, 3 \mathrm{c}, \mathrm{d}, 4 \mathrm{c}, \mathrm{d}$ ) show the time interval of applicability of the asymptotic solution at the porous medium outlet. Depending on the type of particles, the asymptotics is close to a numerical solution for time intervals from 0 to 50200. 


\section{Discussion}

The paper considers the one-dimensional filtration problem of a monodisperse suspension in a porous medium. The suspension displaces pure water from a porous medium with an unevenly distributed initial deposit. In contrast to the standard model, it is assumed that the pores accessibility and the fractional flow of solid particles change as deposit accumulates. Such a problem is close to the filtration problem in an inhomogeneous porous medium [18]. In this case, the velocity of the concentration front depends on the distribution of the initial deposit, and the concentration front of the of suspended particles is curvilinear.

Filtration of the suspension in a porous medium without an initial deposit occurs more intensively near the inlet; the resulting deposit is unevenly distributed and is a decreasing function of the coordinate. If during reverse pumping of pure water, incomplete mobilization of the retained particles is proportional to the retained particles concentration, then the remaining deposit also decreases with increasing $x$. The problem of the periodic change of injection of suspension and reverse flow of water, taking into account the mobilization of particles, is more complex and must be studied separately.

Accounting for the diffusion and dispersion of a suspension flow significantly complicates the filtration model. At present, an explicit analytical solution of such problems has been obtained only for the simplest cases [19].

\section{Conclusions}

An explicit analytical solution of the filtration problem in a porous medium with an initial deposit has been constructed. The line of curvilinear concentration front of suspended particles is obtained in explicit form.

It is shown that the solution of the filtration problem satisfies natural physical conditions: the concentration of retained particles is constant in time before the concentration front and increases in time behind the front; the suspended particles concentration is zero before the front and is positive behind the concentration front.

An asymptotic solution of the filtration problem is constructed behind the concentration front. Numerical calculations show the proximity of the asymptotics to the solution. Depending on the type of suspended particles of the suspension, at the porous medium outlet the acceptable accuracy of the asymptotic solution is maintained until time 50-200.

Analytical solutions of the filtration problem determine in an explicit form the dependence of the suspended and retained particles concentrations on external parameters, giving way to predict the results of experiments and reduce the amount of laboratory research designed to optimize the filtration process [20].

\section{References}

1. Khilar K C and Fogler H S 1998 Migrations of fines in porous media (Dordrecht: Kluwer Academic Publishers) p 172

2. Tsuji M, Kobayashi S, Mikake S, Sato T and Matsui H 2017 Post-Grouting Experiences for Reducing Groundwater Inflow at $500 \mathrm{~m}$ Depth of the Mizunami Underground Research Laboratory Japan Procedia Engineering 191 pp 543-550

3. Chrysikopoulos C V and Syngouna V I 2014 Effect of gravity on colloid transport through water-saturated columns packed with glass beads: modeling and experiments Environmental Science \& Technology 48 pp 6805-6813

4. You Z, Badalyan A and Bedrikovetsky P 2013 Size-Exclusion Colloidal Transport in 
Porous Media-Stochastic Modeling and Experimental Study SPE J. 18(04) pp 620-633

5. Herzig J P, Leclerc D M and Legoff P 1970 Flow of suspensions through porous media - application to deep filtration Industrial and Engineering Chemistry 62 pp 8-35

6. Kuzmina L I, Osipov Yu V and Zheglova Yu G 2018 Analytical model for deep bed filtration with multiple mechanisms of particle capture International Journal of Nonlinear Mechanics $\mathbf{1 0 5}$ pp 242-248

7. Vyazmina E A, Bedrikovetskii P G and Polyanin A D 2007 New classes of exact solutions to nonlinear sets of equations in the theory of filtration and convective mass transfer Theoretical Foundations of Chemical Engineering 41(5) pp 556-564

8. Bedrikovetsky P, You Z, Badalyan A, Osipov Y and Kuzmina L 2017 Analytical model for straining-dominant large-retention depth filtration Chemical Engineering Journal 330 pp 1148-1159

9. Kuzmina L I and Osipov Yu V 2016 Filtration of forward and back flow of suspension in porous media International Journal for Computational Civil and Structural Engineering 12(3) pp 145-150

10. Kuzmina L I, Osipov Yu V and Galaguz Y P 2017 Numerical and asymptotic modeling of a filtration problem with the initial deposit International Journal for Computational Civil and Structural Engineering 13(3) pp 70-76

11. Bedrikovetsky P 2013 Mathematical theory of oil and gas recovery: with applications to ex-USSR oil and gas fields Springer Science \& Business Media 4 p 600

12. Bradford S, Kim H, Haznedaroglu B, Torkzaban S and Walker S 2009 Coupled factors influencing concentration-dependent colloid transport and retention in saturated porous media Environmental Science Technology 43 pp 6996-7002

13. Kuzmina L, Osipov Yu 2018 Deep bed filtration with multiple pore-blocking mechanisms MATEC Web of Conferences 19604003

14. You Z, Osipov Y, Bedrikovetsky P and Kuzmina L 2014 Asymptotic model for deep bed filtration Chemical Engineering Journal 258 pp 374-385

15. Galaguz Yu P 2017 Realization of the TVD-Scheme for a Numerical Solution of the Filtration Problem International Journal for Computational Civil and Structural Engineering 13(2) pp 93-102

16. Galaguz Yu and Safina G 2016 Modeling of Fine Migration in a Porous Medium MATEC Web of Conferences 8603003

17. Toro E F 2009 Riemann solvers and numerical methods for fluid dynamics. 3 ed. Springer, Dordrecht p 724

18. Galaguz Yu and Safina G 2017 Calculation of the filtration in a heterogeneous porous medium MATEC Web of Conferences 11700052

19. Altoe J E, Bedrikovetsky P G, Siqueira A G, de Souza A L and Shecaira F 2006 Correction of Basic Equations for Deep Bed Filtration with Dispersion Journal of Petroleum Science and Engineering $51 \mathrm{pp}$ 68-84

20. Bedrikovetsky P G, Marchesin D, Checaira F, Serra A L and Resende E 2001 Characterization of deep bed filtration system from laboratory pressure drop measurements Journal of Petroleum Science and Engineering 32(3) pp 167-177 